\title{
Equal Area Partition-Based Energy Efficient Routing Algorithm for Circular WSN
}

\author{
Hu Liqin $(\mathbb{D}$, Wang Sanyou $(\mathbb{D}$, Ma Fujun $\mathbb{D}$, and Zhang Shubo \\ Zhejiang College of Construction, Hangzhou 311200, China \\ Correspondence should be addressed to Wang Sanyou; wangsy1025@163.com
}

Received 1 July 2021; Accepted 22 July 2021; Published 10 August 2021

Academic Editor: Jian Su

Copyright @ $2021 \mathrm{Hu}$ Liqin et al. This is an open access article distributed under the Creative Commons Attribution License, which permits unrestricted use, distribution, and reproduction in any medium, provided the original work is properly cited.

\begin{abstract}
Wireless sensor network (WSN) is communicating method with each other without the assistance of communication cable. The circular environment is an important application scenario in WSN. As a core technology, routing algorithm is the brain of WSN monitoring system. In this paper, we propose a new routing algorithm named Equal Area Partition Based Energy Efficient Multipath Algorithm (EAPEEM), which tries to exploit sector equal area model in node deployment, data transfer, and route maintenance. Existing algorithms, as compared to our existing models, do not fully match with routing and nodes. One attractive feature of our protocol is its balanced network transmission capacity and improved node energy utilization, which can reduce the possibility of system damage. Simulations show that EAPEEM can retain at least 28.6\% of batteries and expand stability by more than $43 \%$.
\end{abstract}

\section{Introduction}

Manufacturing costs of sensing components and batteries in wireless sensors have continued to decrease. Sensors with mobility and energy consumption have reduced the difficulty of information monitoring in different fields [1]. The number of wireless devices in various application fields has increased significantly [2-5]. The amount of data generated by sensor nodes will also increase significantly with the number of sensor nodes. This puts forward requirements for resource allocation [6, 7]. At present, the methods of enhancing convergence (communication) are mainly divided into two types: upgrading communication technology and organizing wireless sensor networks. Among them, the main means to upgrade communication technology is to build $4 \mathrm{G}$ or $5 \mathrm{G}$ [8] networks. 5G networks have more powerful mobile bandwidth and more flexible access methods. However, an open wireless access network requires a large number of chips [9-12] to build various types of equipment (high-density base station(BS), etc.). This makes upgrading the communication technology costly and only suitable for a few large cities. Some areas lack $5 \mathrm{G}$ base station construction conditions. WSN is an important technology to meet the harsh conditions and special needs of networking. It combines the large-scale deployment of sensor nodes according to a specific algorithm and cooperates with these nodes to sense [13] and calculate indicators. Therefore, WSN is an indispensable technology. WSN has the advantages of convenient installation, low maintenance cost, and flexible deployment. It has been widely used in construction, forest, marine, and manufacturing [14]. As the brain of WSN, routing algorithm can efficiently combine nodes. Among them, routing algorithms based on information such as node's computing power, energy, and location have become a hot research in WSN.

Therefore, many scholars have conducted research on routing algorithms and published a series of research results. Initially, Heinzelman et al. [15] proposed the LEACH algorithm. The node information is first transmitted to the cluster head $(\mathrm{CH})$ of the cluster where it is located and then uniformly sent by $\mathrm{CH}$.

However, task intensity of $\mathrm{CH}$ is much greater than that of ordinary nodes. SEP enhances the robustness of the network by increasing the energy of some nodes [16]. For energy and data needs, many new studies have been published in recent years on reducing power consumption, 
improving data transmission, and extending network life cycle [17]. Obviously, routing algorithms are an important part of WSN. In this section, we reviewed the latest routing algorithms. Yi et al. proposed HEER in 2016. The greedy algorithm was utilized to build routing path. The characteristic of HEER is that there is no need for cluster reorganization [18], as for network throughput and packet delivery ratio. To solve inconvenience by energy-efficient and robust multipath [19], the Cluster Aided Multipath Routing algorithm (CAMP) outperforms classic algorithms (such as LEACH and DEEC). CAMP displays more prominent performance of living, timing, and coverage ratio because of balancing batteries of link [20]. Another new algorithm is VCH-ECCR [21]. The routing data between different vice cluster heads was obtained. Longevity of the network was enhanced. Vinitha describe a cat-salp swarm algorithm (ClogSA) based on optimal selection of the multipath. Its core is that choosing the optimal hops in progressing the routing, which reduce the energy constraints. The results showed that $C \log S A$ effectively improved the link lifetime and reaction speed [22].

However, the above research results mainly focus on improving the indicators of cluster head, routing, and data volume. Based on their research analogies, most of them are classic algorithms, and the research background of the above-mentioned research is generally a square space. However, the circular WSN has the broadest application prospects. There are two main reasons for this. First of all, in extreme environments, nodes are usually deployed (tossed) randomly, and the deployment area is closer to a circle. Secondly, the circle is easier to match with the sensor perception model. However, it is difficult for the traditional transmission strategy improvement and cluster strategy improvement to make a major breakthrough in the circular area. Based on coverage rate, premature node decay becomes an important issue in circular communication systems.

In order to build a routing algorithm that is more suitable for a circular area, this paper combines the equal area partition model and the $m$-layer multihop routing, and conducts theoretical derivation. Therefore, this paper proposes Equal Area Partition-Based Energy Efficient Multipath Algorithm (EAPEEM). The EAPEEM algorithm boost the life cycle of the network in the application of the circular area, balance the network energy consumption, and enhance the data throughput capacity.

The rest of this paper is arranged as follows: introduction is presented in Section 1. Characteristic of circular WSN analysis and problems are introduced in Section 2. The proposed algorithm is described in Section 3. In Section 4, the simulation and results are discussed. Finally, conclusions are summarized in Section 5.

\section{Characteristic Analysis of Circular WSN}

2.1. Circular Applicability and Characteristic Analysis. In the application, the circular area can meet more actual scenes. Especially when the sensor node needs to be thrown by an airplane, in this case, the monitoring area formed by the sensor is closer to a circle. Circular WSN mainly implements

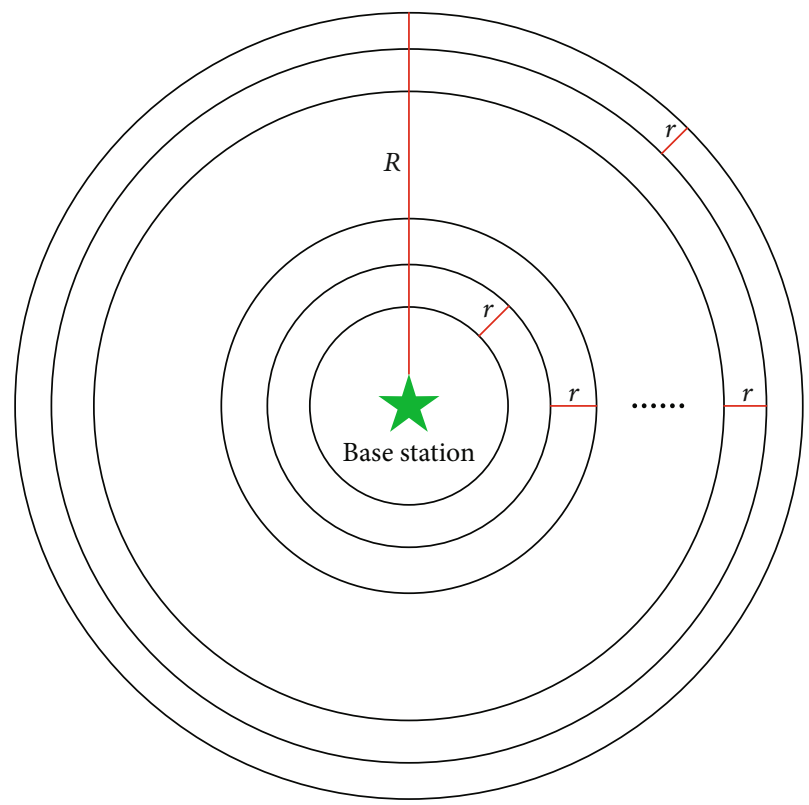

FIGURE 1: Equidistant concentric circle model.

monitoring data collection and transmission tasks in the area. Circular WSN mainly implements monitoring transmission tasks. As a major unit of a complete automated monitoring network. This makes high-performance routing protocols one of the key factors for the stable operation of the monitoring system.

Considering the difference between a circular area and a square area, there are many differences in deploying WSN in a circular area compared to deployment in other environments. These differences cause the routing protocol based on the square area to not be directly copied to the application of the circular WSN. Therefore, these characteristics of the circular area will become the main factors limiting the performance of the protocol when designing the routing protocol. Through analysis, the main similarities and differences of the circular area are as follows:

(1) In the circular area, the location of nodes is only related to latitude [23]

(2) There is a difference between the circular area and the square area in the effective coverage in the edge area

2.2. Comparison of Traditional Methods. The existing research results of circular WSN mainly focus on circular area decomposition and information transmission methods. They usually adopt the equidistant concentric circle model (as shown in Figure 1). In the equidistant concentric circle model, $r$ is the distance between adjacent concentric circle. On this basis, the existing research also considers two transmission modes, single-hop and equidistant multihop. This section analyzes the characteristics of these two transmission modes in the equidistant concentric circle model. The main analysis focuses on two aspects: information transmission distance and unit information density (hole problem). 


\subsection{Equidistant Concentric Circle Single-Hop Transmission} Model. Based on the equidistant concentric circle singlehop transmission model, the information transmission path in the monitoring area is node to $\mathrm{CH}$ to BS. This model is greatly affected by the distance (as shown in Figure 2).

For the circular area of WSN used in Figure 2, the major parameter to consider (in 1 round) is the amount of data. For equidistant concentric circle single-hop transmission model, there are some assumptions. $N_{S}$ nodes are uniformly deployed in the network, the probability of cluster selection is $P_{S}$, the amount of data collected by each node in each round is $D_{S}$, the radius of the tested area is $R$, and it is divided into $K$ layers of concentric circles. The distance between node $i$ and BS is $R_{i}$. The data transfer load in the network (in 1 round) can be defined as the product of the amount of data and the transfer distance:

$$
\sum_{i=0}^{K} R_{i} \frac{R_{i}^{2}-R_{i-1}^{2}}{R^{2}} P_{S} N_{S} D_{S} .
$$

This method has two obvious characteristics. First, the transfer distance of data (in 1 round) is positively correlated with $R$ and $K$. Secondly, the life of the outer ring $\mathrm{CH}$ is shorter, which will affect the effective coverage of the circular area.

\subsection{Equidistant Concentric Circle Multihop Transmission} Model. In the equidistant concentric circle single-hop model (the short life of the outer ring $\mathrm{CH}$ causes the effective coverage of the circle), some studies use multihop transmission to reduce the impact of distance. Multihop can reduce the forwarding distance of the cluster head in the outer circle and extend the effective coverage time. In the equidistant concentric circle multihop transmission model, the data transmission path in the monitoring area is node to $\mathrm{CH}$ to multiple $\mathrm{CHs}$ to BS. It is characterized in that the data collected by the node is sent to the BS through multiple CHs. Based on the uniform deployment of nodes, this method is greatly affected by the multihop strategy (as shown in Figure 3).

$N_{m}$ nodes are uniformly deployed in the network, the probability of cluster selection is $P_{m}$, the amount of data collected by each node in each round is $D_{m}$, the radius of the tested area is $R$, and it is divided into $K$ layers of concentric circles. The distance between node $\mathrm{j}$ and BS is $R_{j}$. The data transfer load in the network (in 1 round) can be defined as the product of the amount of data and the transfer distance:

$$
\sum_{j=0}^{K} j r \frac{R_{j}^{2}-R_{j-1}^{2}}{R^{2}} P_{m} N_{m} D_{m} .
$$

Comparing formula (1) and formula (2), the probability of selection and the amount of data collected per unit time of the node are the same. The difference between the two transfer loads is reflected in the difference in transfer distance, that is, the comparison of $R i$ and $j r$. Normally, most of $R i$ in a single jump is greater than $d 0$

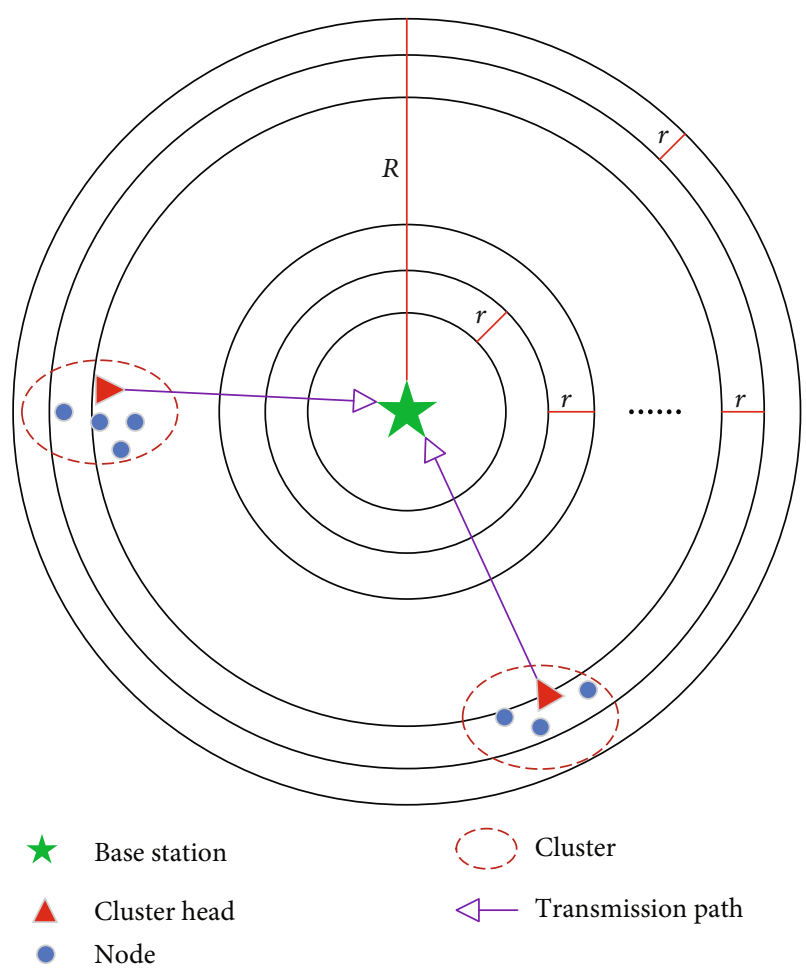

FIgURE 2: Equidistant concentric circle single-hop transmission model.

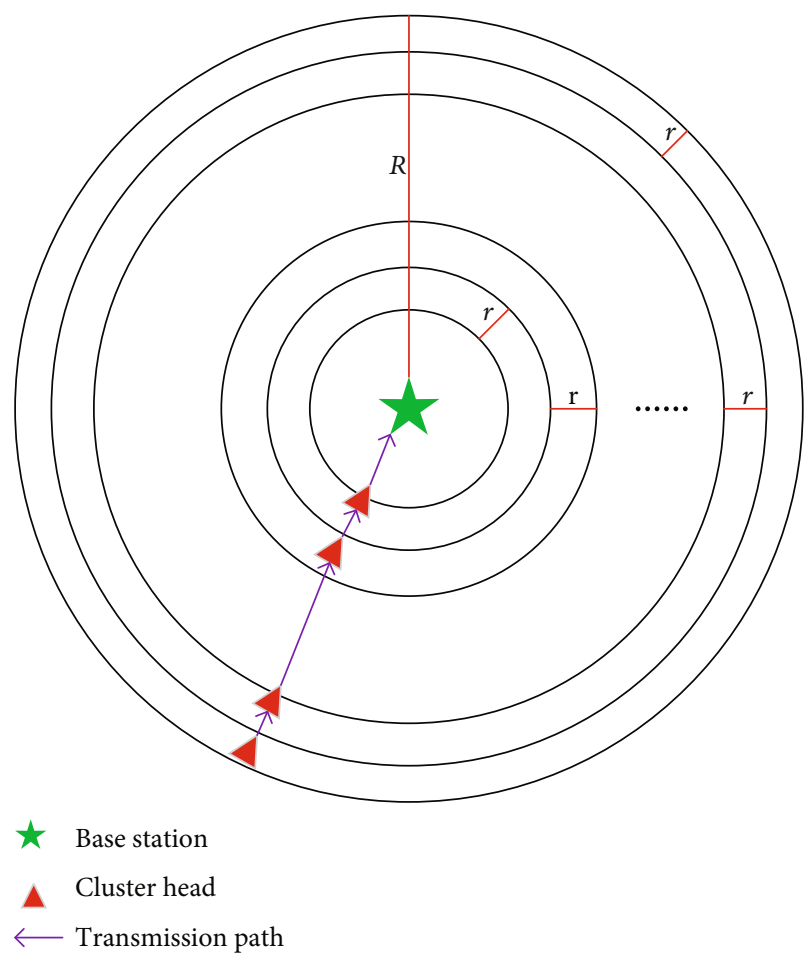

Figure 3: Equidistant concentric circle multihop transmission model.

(explained in Section 2.2), and the distance causes huge pressure on information transmission. In the case of equidistant multiple hops, the relationship between $r$ and $d 0$ is more 
complicated [24]. Therefore, for a circular WSN, the equidistant concentric circle model combined with a single hop increases the coverage pressure of edge nodes. The equidistant concentric circle model combined with equidistant multiple hops will make the nodes close to the BS take on the heavy transfer task.

\section{EAPEEM Routing Algorithm}

Section 2.2 analyzes the shortcomings of routing algorithm in equidistant concentric circle. We propose the EAPEEM routing algorithm. EAPEEM improves the basic model, cluster size, and routing method. EAPEEM can transmit data in large-scale circular and quasicircular spaces. The EAPEEM algorithm is mainly composed of four stages: (a) the construction of a sector equal area model (area and node heterogeneity); (b) heterogeneous node deployment; (c) cluster establishment; (d) routing establishment. First, the sector equal area model structure is proposed. Based on the equal area division of the angle and fan-shaped areas, the nodes are divided into normal and edge nodes according to the energy level and position end information. Then, node deployment includes a combination of specific node deployment and free random deployment. In the stage of cluster establishment, the clustering strategy is optimized. In the routing establishment stage, EAPEEM delineates the coverage of edge nodes and improves the $m$-multiple hop strategy for equal area areas.

3.1. Network Model. The monitoring target object of a sector equal area WSN is a circular or quasicircular area. This paper abstracts the space structure studied into a circle, and information is transmitted in this circular area according to certain rules. Energy will be consumed in the process of WSN information transmission. With the extension of the transmission distance, the energy consumed by the communication between nodes will be more. Nodes far away from the BS and nodes that forward multiple times may have a series of problems such as inability to maintain stable transmission. Therefore, based on the characteristics of the circle, a WSN model with the following characteristics is proposed:

(a) The node does not have two positions in WSN, and no existing nodes are added or removed

(b) The node uses a Boolean perception model. The sensing range and communication range of the node are both ideally circular

(c) There is one and only one BS in a single monitoring area. The BS has enough performance and is not included in the network consumption [25]

(d) All wireless devices in the area can communicate with each other and have the ability to send information to the BS [26]

(e) Network needs to consider some redundancy [7, 27]

Formula (3) and formula (4) show the calculation method of energy consumption.
The formula for sender:

$$
E_{T X}= \begin{cases}e \times E_{f s} \times d^{2}+e E_{p}, & d<d_{0}, \\ e \times E_{m p} \times d^{4}+e E_{p}, & d \geq d_{0} .\end{cases}
$$

The formula for receiver:

$$
E_{R X}=e E_{p}
$$

In the formula, $e$ represents the amount of information; $E_{p}$ is transceiver circuit consumption; $d$ is the distance; $d_{0}$ is the threshold under the free space model and the multipath model and $d_{0}=\left(E_{f s} / E_{m p}\right)^{1 / 2} ; E_{f s}$ and $E_{m p}$ represent two factors for calculation, respectively.

3.2. Sector Equal Area Model. There are possible problems of the equidistant concentric circle model in WSN applications. When single-hop communication is used, coverage holes are prone to appear at the edge of the monitoring area. Under the isometric multihop strategy, the area close to the BS is likely to form an "energy hole." In this case, the area of nodes deployed between adjacent rings in the isometric concentric circle model gradually decreases nonlinearly. That is to say, the number of nodes between two adjacent rings also shows a nonlinear decrease (as shown in Figure 4). In the figure, Deployment area $1>$ Deployment area $2>$ Deployment area 3 . The number of nodes in the three areas also decreases sequentially, resulting in different communication pressures between two adjacent rings.

Therefore, in order to balance the communication load in different areas. The sector equal area model is shown in Figure 5. Among them, the radius is $R$, and angle of sector area is $\theta$. In the sector with an angle of $\theta$, except for the arc-shaped area $S_{0}$ near the monitoring edge, the remaining part is divided into $V$ parts of equal area, including $V-1$ trapezoids of equal area and 1 triangle, denoted as $S_{1}=S_{2}=\cdots=S_{V}$. Then, the sector area $S_{\theta}$ is:

$$
S_{\theta}=\frac{\theta R^{2}}{2}
$$

Excluding the edge of the arc-shaped area $S_{0}$, the remaining area $S_{\triangle}$ is:

$$
S_{\triangle}=\frac{1}{2} \times 2 R \sin \frac{\theta}{2} \times R \cos \frac{\theta}{2}=R^{2} \sin \frac{\theta}{2} \cos \frac{\theta}{2} .
$$

Divide the triangular area into $V$ layers; the area of a single layer is:

$$
S_{1}=\cdots=S_{V}=\frac{S_{\triangle}}{V}=\frac{R^{2} \sin \theta}{2 V} .
$$




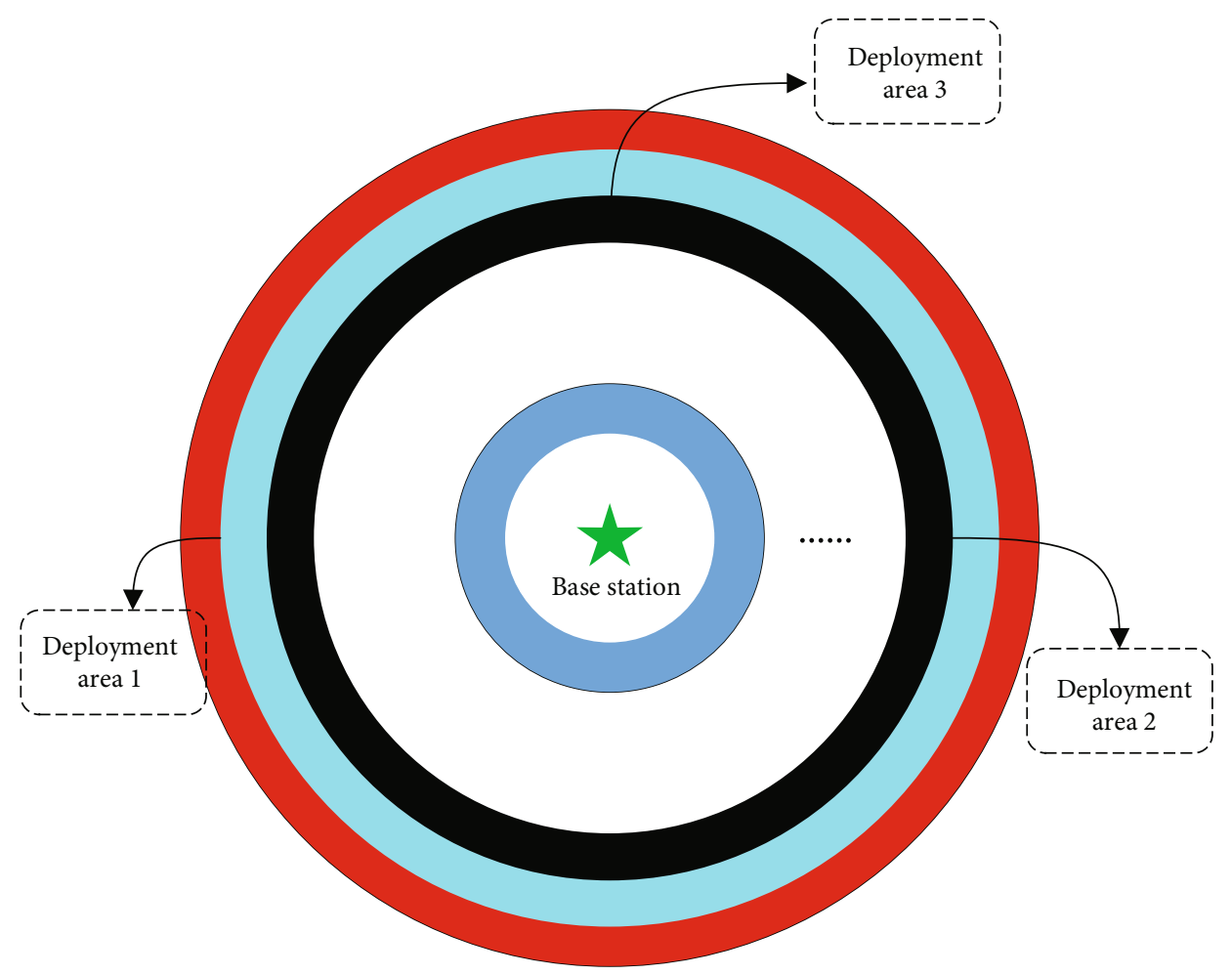

FIgURE 4: Comparison of the deployment area of adjacent rings.

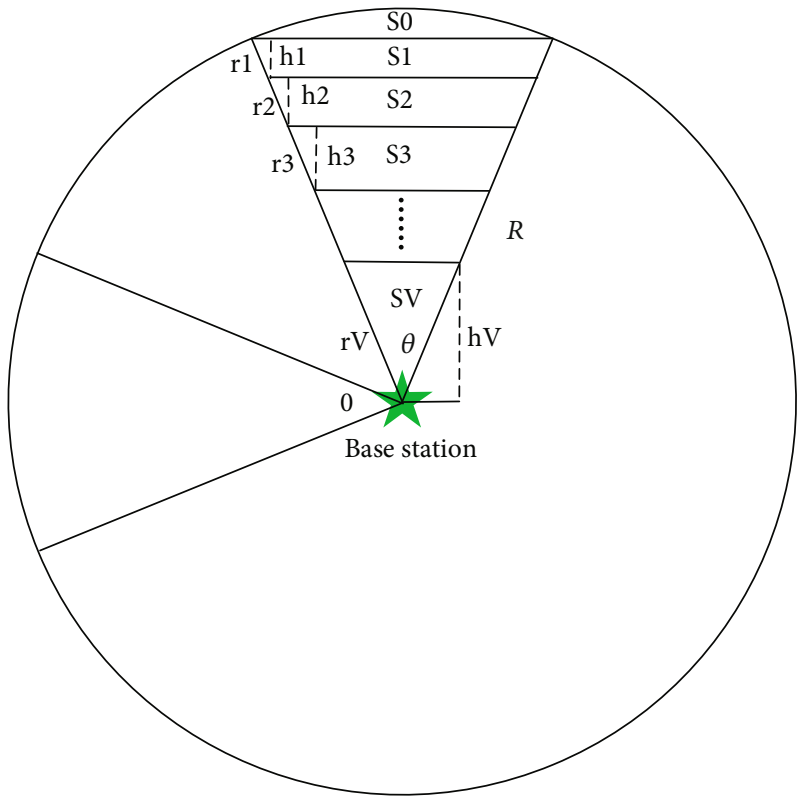

Figure 5: Sector equal area model.

The triangular area with the area equally divided is shown in Figure 6. Then, it can be deduced that the side length $r_{V}$ of the isosceles triangle area $S_{V}$ is:

$$
\frac{R^{2} \sin \theta}{2 V}=\frac{1}{2} \sin r_{V}^{2} \longrightarrow r_{V}=\sqrt{\frac{R^{2}}{V}}
$$

From the above formula, the high $h_{V}$ and $t_{V}$ of the $S_{V}$ area can be derived as:

$$
h_{V}=r_{V} \cos \frac{\theta}{2}=\sqrt{\frac{R^{2}}{V}} \cos \frac{\theta}{2} .
$$

Due to $S_{1}=S_{2}=\cdots=S_{V}$, the base and height of the triangle $S_{V}$ can be derived (as in formula (10)). According to the same area of each block, the general formula of the relationship between $t$ and $h$ can be derived from the relationship between $t_{1}, t_{2}$, and $t_{3}$ :

$$
\left\{\begin{array}{l}
t_{1}=t_{2}+2 h_{1} \tan \frac{\theta}{2} \\
t_{1}=t 3+2 h_{2} \tan \frac{\theta}{2}+2 h_{1} \tan \frac{\theta}{2}
\end{array} \longrightarrow t_{i}=t_{j}+2\left(h_{j-1}+\cdots+h_{i}\right) \tan \frac{\theta}{2} .\right.
$$

From formula (10), it can be concluded that there is no exponential (rising or falling) trend between the two most relevant parameters of the same area division, $t$ and $h$. Therefore, the sector equal area model can effectively alleviate exponential relationship between adjacent rings in the concentric circle model.

3.3. Node Heterogeneous. Based on the CPU's ability to uniformly process the collected information, location complexity and sensor diversity will cause extremely uneven energy consumption of sensor nodes; WSN will also show the characteristics of energy heterogeneity. As analyzed in Section 2.2, boundary node dies faster than other locations. 


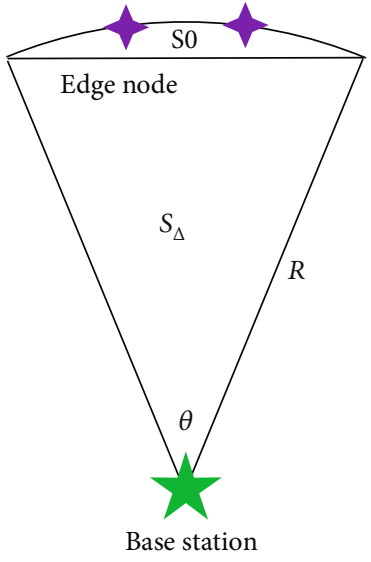

Figure 6: Node heterogeneous diagram.

Therefore, to improve the energy coverage of the $S_{0}$ area, this method can increase the working time of the nodes in the edge area $S_{0}$ of the circular WSN.

According to the description in Section 3.2, there are $S_{0} \sim S_{V}$ areas in a sector with an angle of $\theta$. In order to balance the deployment density of adjacent regions in the sector, make $S_{1}=S_{2}=\cdots=S_{V}$. And $S_{0}$ is located at the edge of the monitoring range and $S_{0}$ does not use the sector equal area model. Therefore, this algorithm adopts heterogeneous nodes. After the information form is unified, energy becomes the main factor limiting the comprehensive performance of WSN. Based on the existence of two regions $S_{0}$ and $S_{\triangle}$ in Figure 7, nodes contain the normal and the edge. First, the edge nodes save more initial energy, and they can complete information collection and long-distance information transmission. The number of high-energy edge nodes is much smaller than that of normal nodes. By considering the area of $S_{0}$ and $S_{\triangle}$, the number and energy of normal and edge are obtained. In a sector with an angle of $\theta$, the proportion of the area equally divided area is:

$$
\frac{S_{\triangle}}{S_{\theta}}=\frac{(1 / 2) R^{2} \sin \theta}{(\theta / 2 \pi) R^{2} \pi}=\frac{\sin \theta}{\theta} .
$$

Because the edge of the monitoring area needs stronger energy coverage, location of the edge is $S_{0}$ (Figure 6). This kind of node heterogeneous strategy can intuitively reflect the partition characteristics of the sector equal area model, making the average energy of the nodes in the edge work area higher, which meets the requirements of "direct" transmission. Moreover, this heterogeneous strategy also meets the requirements of intercluster routing below, enabling it to solve the "energy hole" problem caused by multihop transmission and enhance the coverage of border areas. So the number of edges is $2 \pi / \theta$, and the energy coefficient is $(1+(\sin \theta / \theta))$.

3.4. Clustering Strategy. In Sections 3.2 and 3.3 of this paper, we have discussed the issue of sector equal area model and node heterogeneity. The selection of cluster heads in areas is as $S_{1}=S_{2}=\cdots=S_{V}$. This section discusses the clustering strategy.

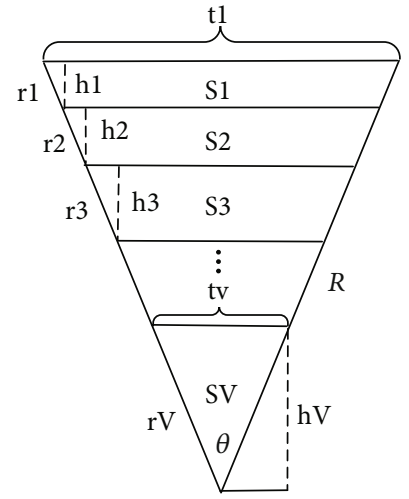

Figure 7: Schematic diagram of area equalization.

The circular deployment area can be abstracted into a circular space with a radius of $R$ with $N$ nodes. Then, make the following assumption: based on uniform clustering, the normal nodes in the trapezoidal or triangular area of the same area in each layer contains $C$ clusters. Processing of information transmission, the cluster head receives the signals transmitted by the members of the cluster and sends the information to other cluster heads or base stations after processing.

A sector is with an angle of $\theta$. In a sector with an angle of $\theta$, the total number of the normal is $\theta N / 2 \pi$, which in a single layer of an equal area is $(N \sin \theta) /(2 \pi V)$.

Then, under the premise of uniform deployment, the sensing distribution of each cluster is shown in formula (12).

$$
\varphi=\frac{N \sin \theta}{2 \pi C V r_{c}^{2}} .
$$

In formula (13), $N$ represents the normal number in the circular area, and $r_{c}$ is radius of each subunit. Then, the range of $r_{c}$ is $[0,(2 R / V)]$. The expected $d_{E}$ is calculated as:

$$
d_{E}=\int_{\theta=0}^{2 \pi} \int_{r_{C}=0}^{2 R / V} \varphi r_{C}^{3} d r_{C} d \theta=\frac{R^{2} N \sin \theta}{2 C V^{3}} .
$$

For the normal node, the normal node only needs to transfer information to $\mathrm{CH}$. The intracluster communication distance is less than the threshold $d 0$, and the intracluster communication uses single-hop transmission. That is, the energy used by a single normal node (non- $\mathrm{CH}$ node) to construct a WSN can be described as formula (14), and $E_{p}$ is the circuit consumption:

$$
E_{\text {normal }}=\varepsilon_{f s} d_{E}^{2}+E_{p} .
$$

For CH nodes: $E_{C H}=E_{R X}+E_{T X}$

$$
E_{C H}=E_{R X}+E_{T X}=\left(\frac{N \sin \theta}{C V}-1\right) E_{p}+E_{p}+\varepsilon_{f s} d_{E}^{2} .
$$


For clusters, the single-hop transmission and forwarding consumption of $\mathrm{CH}$ node are shown in formula (16).

$$
\begin{aligned}
E_{\text {cluster }}= & E_{C H}+\left(\frac{N \sin \theta}{C V}-1\right) \varepsilon_{f s}\left(\frac{R^{2} N \sin \theta}{2 C V^{3}}\right)^{2} \\
& +\left(\frac{N \sin \theta}{C V}-1\right) E_{p} .
\end{aligned}
$$

It is worth noting that in formula (17), $d_{t}$ is a fluctuation interval, which is estimated by expectation. A point is randomly selected in the circle, and the expectation of the distance from this point to the center of the circle is $d_{t}=2 / 3 R$. It can be obtained that $\mathrm{CH}$ forwarded the energy consumption $E_{t}$ :

$$
E_{t}=C V \frac{2 \pi}{\theta} d_{t}^{4} \varepsilon_{f s}
$$

Total energy consumption of the entire network $E_{\text {total }}$.

$$
E_{\text {total }}=C V \frac{2 \pi}{\theta} E_{\text {cluster }}+E_{t}
$$

The relationship between $V$ and $N, C$ and can be obtained from $E_{\text {total }} \cdot V=\left(\left(9 N^{3} \sin ^{3} \theta\right) / 8 C^{2}\right)^{1 / 4}$.

3.5. Routing Determination. In the data transfer process of the routing algorithm, all nodes in the cluster need to transfer information to the $\mathrm{CH}$ first and then transfer the information to the BS from the $\mathrm{CH}$. This process needs to consider the issue of information transmission distance and $\mathrm{CH}$ energy consumption. Therefore, the EAPEEM algorithm has made improvements in the routing method. Based on the heterogeneous node design proposed in Section 3.3 of this paper, the routing determination in this paper is divided into two categories. The edge node $R$ away from the $\mathrm{BS}$ serves as the $\mathrm{CH}$ and directly communicates with the BS. In the sector equal area model area, $m$ multiple hops are used in Figure 8.

To balance consumption and $\mathrm{CH}$ load to the greatest extent, multihop routing with equal area and $m$ multiples is proposed. Each information transmission needs to span adjacent $m-1$ equal-area spaces if link from BS is less than $m-1$ space.

\section{Simulation and Discussion}

4.1. Simulation Parameters. This article uses MATLAB as the simulation platform (Table 1). We have made improvements for the shortcomings of multihop and edge areas and proposed the EAPEEM. This part conducts results on EAPE EM and compares to LEACH, SEP, and CZSEP algorithms in terms of wireless sensor network survival time, number of clusters, and data throughput.

4.2. Performance Analysis. In the same experimental environment, the LEACH, SEP, CZSEP, and EAPEEM are simulated.

Figure 9 is a comparison diagram of the dead node trend of four algorithms such as LEACH, SEP, CZSEP, and EAPE $\mathrm{EM}$ at the same time. Except for LEACH and SEP, the other

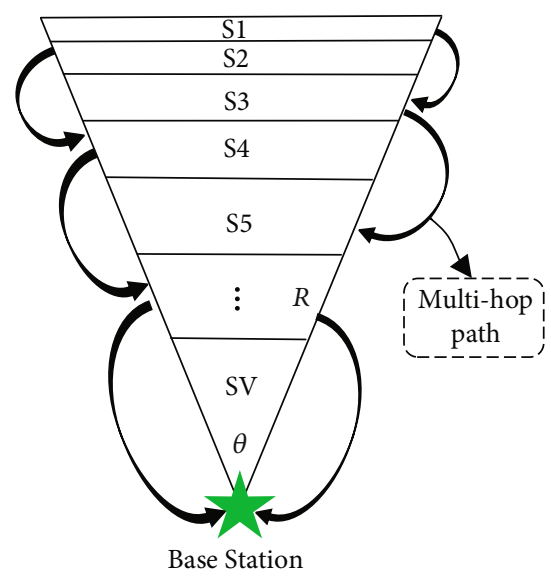

FIGURE 8: Routing strategy.

TABle 1: Parameters in computing.

\begin{tabular}{lc}
\hline Type & Value \\
\hline Sensors & 100 \\
Network radius & $100 \mathrm{~m}$ \\
BS location & Center of a circle \\
Initial node energy (normal) & $0.5 \mathrm{~J}$ \\
$\varepsilon_{f s}$ & $0.0013 \mathrm{pJ} / \mathrm{bit} / \mathrm{m}^{4}$ \\
$\varepsilon_{m p}$ & $10 \mathrm{pJ} / \mathrm{bit} / \mathrm{m}^{2}$ \\
$E_{p}$ & $50 \mathrm{~nJ}$ \\
\hline
\end{tabular}

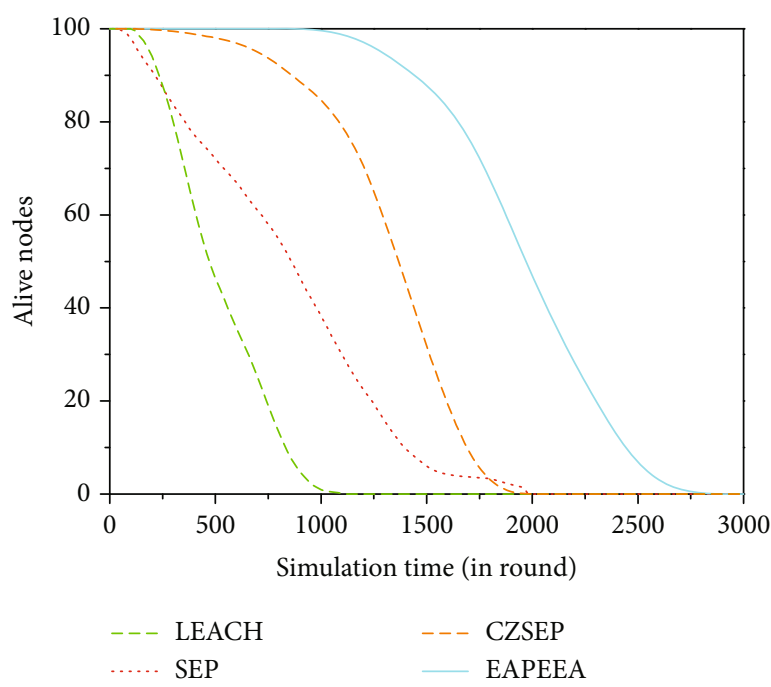

Figure 9: Comparison of network life.

two algorithms have no obvious downward trend in the no surviving nodes before the first 750 rounds. However, LEACH and SEP had obvious node deaths in early time. After that, the first dead nodes of SEP and CZSEP all appeared before the 150th round. However, the downward trend of CZSEP is not obvious. After EAPEEM rounds 1080 , nodes began to die in large numbers. The first dead 


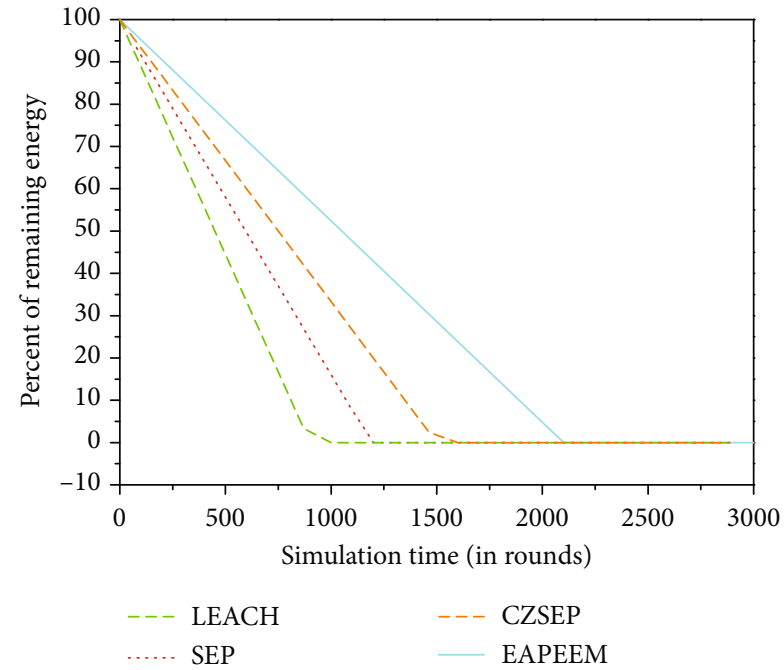

Figure 10: Percentage of remaining energy.

node appearing is delayed by at least $86.7 \%$ in EAPEEM. Therefore, the EAPEEM algorithm effectively delays the appearance of the first dead node. In addition, as CZSEP, low-energy nodes have limited energy but take on important tasks, and dead nodes appear very fast. By contrast, in terms of emergence for dead nodes, after the first dead node appears, the slopes of LEACH and SEP curves are larger. The CZSEP algorithm continues a longer life cycle. But compared with them, the life cycle of EAPEEM algorithm is increased by $43 \% \sim 155 \%$, which means that the network integrity time of EAPEEM algorithm greatly exceeds that of other algorithms. Therefore, the EAPEEM algorithm is significantly better than others of network integrity when the first dead node appears.

Figure 10 is the comparison of average energy consumption. With the other three algorithms, LEACH has the fastest energy consumption. SEP and CZSEP use two-level and three-level heterogeneity, respectively, to reduce energy consumption. But it cannot reach the level of the EAPEEM algorithm. When CZSEP energy is exhausted, EAPEEM still retains $28.6 \%$ of the energy. This shows that the EAPEEM algorithm uses the sector equal area model and $m$-multihop to achieve the effect of network energy balance.

Figure 11 shows the clustering ability. The graph histogram describes the average degree of clustering in equal time. From Figure 11, the cluster of EAPEEM during the experiment is the largest and has the best information collection capabilities (this can also be seen in the data throughput of Figure 12). It is worth noting that SEP and LEACH have dropped significantly. Among them, when LEACH is 1000 round, the network energy consumption is close to exhaustion. SEP no longer has heterogeneous advantages. CZSEP makes the number of $\mathrm{CHs}$ drop evenly by certain strategy. The clusters of EAPEEM is always the best. The EAPEEM algorithm ensures the effectiveness of node data transmission and fully and effectively alleviates the problem of premature death of cluster head nodes due to excessive load. The reason is that the EAPEEM adopts sector equal area model, which

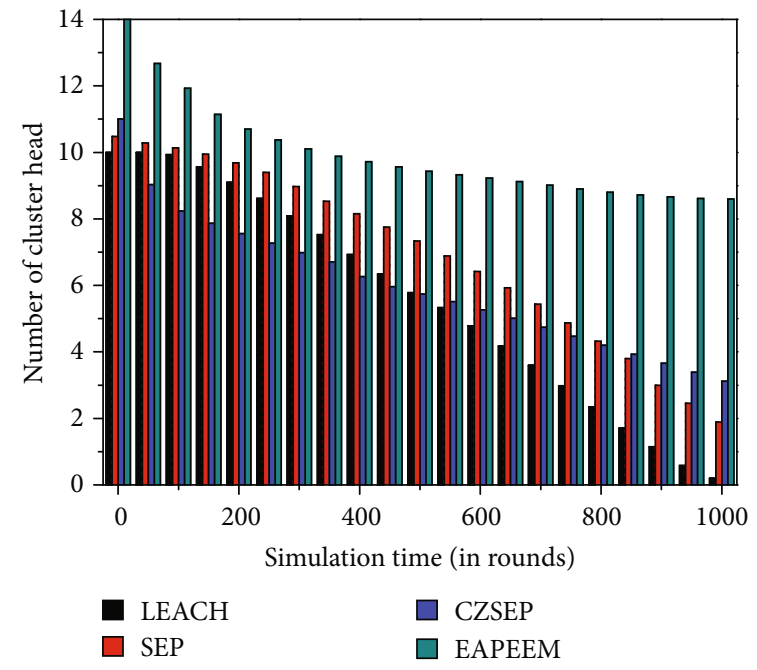

FIGURE 11: Comparison of cluster heads.

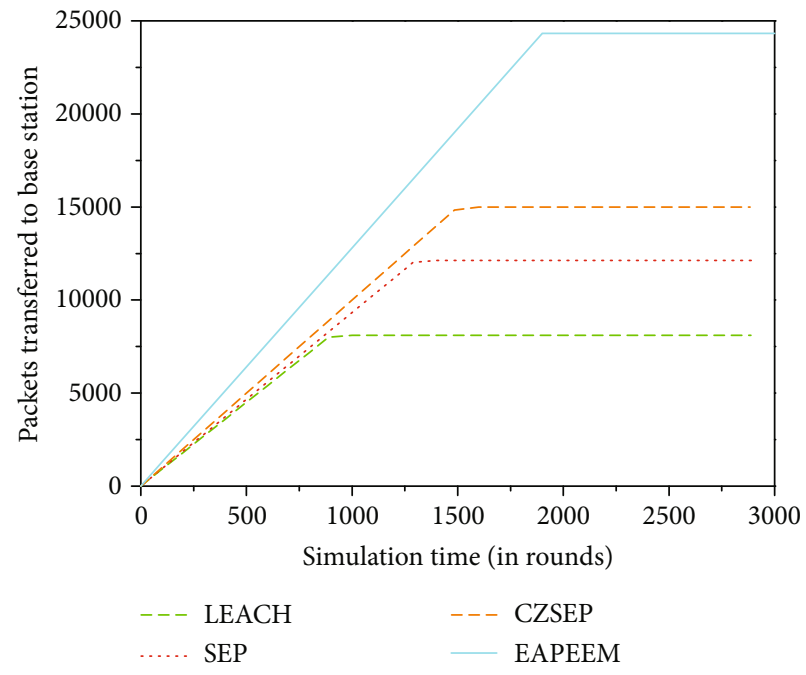

FIGURE 12: Base station receiving data.

makes the area division of cluster heads more reasonable and the network energy consumption is more balanced.

Figure 12 shows the changes of the four algorithms on the total amount of data received by BS. From Figure 12 that LEACH's data throughput capacity is relatively weak, only $33.17 \%$ of EAPPEM and SEP heterogeneous nodes can improve data transmission capacity in a circular area, and the total amount of data is $149.74 \%$ of LEACH. And CZSEP has a unit time data throughput capacity similar to SEP, but has a longer life cycle. And EAPEEM has the strongest unit information collection ability and the longest life cycle. In the experiment, the total throughput of EAPEEM is $162.13 \%$ of the total throughput of CZSEP, which is consistent with the clustering situation in Figure 12.

\section{Conclusion}

Aiming at the edge area coverage problem and the BS area premature aging problem in the concentric circle WSN 
model, This paper proposes the EAPPEM algorithm based on the amount of information generated per unit area. The algorithm introduces the edge to supplement the coverage of the edge area and balances the node density by equal area equalization. In addition, the routing mechanism is improved under the comprehensive consideration of node characteristics and spatial distribution, to avoid local optimal early (problem of premature death of nodes). The goal of EAPPEM is to reduce energy consumption, extend network life, and improve data throughput. Of course, these improvements increase the load of facilities. For future work, reducing the complexity of algorithm will maintain function of system.

\section{Data Availability}

The data that support the findings of this study are available from the corresponding author upon reasonable request.

\section{Conflicts of Interest}

The authors declared no potential conflicts of interest with respect to the research, authorship, and/or publication of this article.

\section{Acknowledgments}

This research is supported by the Zhejiang Province Public Welfare Project of China under grant no. LGF18F030005, Visiting Engineer School-Enterprise Cooperation Project of Colleges and Universities Zhejiang Province of China under grant no. FG2019115, and Basic Scientific Research Project of Zhejiang Construction Vocational and Technical College of China under grant no. Z201910.

\section{References}

[1] M. M. Baig, H. Gholamhosseini, and M. J. Connolly, "Mobile healthcare applications: system design review, critical issues and challenges," Australasian Physical \& Engineering Sciences in Medicine, vol. 38, no. 1, pp. 23-38, 2015.

[2] J. Su, A. X. Liu, Z. Sheng, and Y. Chen, "A partitioning approach to RFID identification," IEEE/ACM Transactions on Networking, vol. 28, no. 5, pp. 2160-2173, 2020.

[3] J. Su, Z. Sheng, A. X. Liu, Z. Fu, and C. Huang, "An efficient missing tag identification approach in RFID collisions," IEEE Transactions on Mobile Computing, pp. 1-12, 2021.

[4] J. Su, Z. Sheng, V. C. M. Leung, and Y. Chen, "Energy efficient tag identification algorithms for RFID: survey, motivation and new design," IEEE Wireless Communications, vol. 26, no. 3, pp. 118-124, 2019.

[5] J. Su, Y. Chen, Z. Sheng, and L. Sun, "Bit query based M-ary tree protocol for RFID tags identification," in GLOBECOM 2017 - 2017 IEEE Global Communications Conference, pp. 17, Singapore, 2017.

[6] Z. Kuang, G. Li, L. Zhang, H. Zhou, C. Li, and A. Liu, "Energy efficient mode selection, base station selection and resource allocation algorithm in D2D heterogeneous networks," Peerto-Peer Networking and Applications, vol. 13, no. 5, pp. 18141829, 2020.
[7] Z. Kuang, L. Zhang, and L. Zhao, "Energy- and spectralefficiency tradeoff with \$\alpha-fairness in energy harvesting D2D communication," IEEE Transactions on Vehicular Technology, vol. 69, no. 9, pp. 9972-9983, 2020.

[8] A. Gupta and R. Jha, "A survey of 5G network: architecture and emerging technologies," IEEE Access, vol. 3, pp. 12061232, 2015.

[9] J. Su, Z. Sheng, A. X. Liu, Y. Han, and Y. Chen, "Capture-aware identification of mobile RFID tags with unreliable channels," IEEE Transactions on Mobile Computing, pp. 1-14, 2020.

[10] J. Su, Y. Chen, Z. Sheng, Z. Huang, and A. X. Liu, "From M-ary query to bit query: a new strategy for efficient large-scale RFID identification," IEEE Transactions on Communications, vol. 68, no. 4, pp. 2381-2393, 2020.

[11] J. Su, Z. Sheng, A. Liu, Y. Han, and Y. Chen, “A group-based binary splitting algorithm for UHF RFID anti-collision systems," IEEE Transactions on Communications, vol. 68, no. 2, pp. 998-1012, 2020.

[12] J. Su, X. Zhao, D. Hong, Z. Luo, and H. Chen, "Q-value finegrained adjustment based RFID anti-collision algorithm," IEICE Transactions on Communications, vol. E99.B, no. 7, pp. 1593-1598, 2016.

[13] J. Su, Z. Sheng, A. Liu, Z. Fu, and Y. Chen, "A time and energy saving based frame adjustment strategy (TES-FAS) tag identification algorithm for UHF RFID systems," IEEE Transactions on Wireless Communications, vol. 19, no. 5, pp. 2974-2986, 2020.

[14] A. Alchihabi, A. Dervis, E. Ever, and F. al-Turjman, "A generic framework for optimizing performance metrics by tuning parameters of clustering protocols in WSNs," Wireless Networks, vol. 25, no. 3, pp. 1031-1046, 2019.

[15] M. Radhika and P. Sivakumar, "Energy optimized micro genetic algorithm based LEACH protocol for WSN," Wireless Networks, vol. 27, article 1665, pp. 1-14, 2021.

[16] S. K. Bhoi, D. Puthal, P. M. Khilar, J. J. P. C. Rodrigues, S. K. Panda, and L. T. Yang, "Adaptive routing protocol for urban vehicular networks to support sellers and buyers on wheels," Computer Networks, vol. 142, pp. 168-178, 2018.

[17] S. Singh, P. Kumar, and J. Singh, "A survey on successors of LEACH protocol,” IEEE Access, vol. 5, pp. 4298-4328, 2017.

[18] D. Yi and H. Yang, "HEER - a delay-aware and energy-efficient routing protocol for wireless sensor networks," Computer Networks, vol. 104, pp. 155-173, 2016.

[19] K. Singh and S. Moh, "An energy-efficient and robust multipath routing protocol for cognitive radio ad hoc networks," Sensors, vol. 17, no. 9, p. 2027, 2017.

[20] M. Sajwan, D. Gosain, and A. K. Sharma, "CAMP: cluster aided multi-path routing protocol for wireless sensor networks," Wireless Networks, vol. 25, no. 5, pp. 2603-2620, 2019.

[21] P. Rohit and S. Deepti, "VCH-ECCR: a centralized routing protocol for wireless sensor networks," Journal of Sensors, vol. 2017, Article ID 8946576, 10 pages, 2017.

[22] A. Vinitha, M. Rukmini, and D. Sunehra, "Energy-efficient multihop routing in WSN using the hybrid optimization algorithm," International Journal of Communication Systems, vol. 33, article e4440, no. 12, 2020.

[23] X. Liu, J. Zhang, S. Jiang et al., “Accurate localization of tagged objects using mobile RFID-augmented robots," IEEE Transactions on Mobile Computing, vol. 20, no. 4, pp. 1273-1284, 2021. 
[24] Y. Huang and Y. Hua, "On energy for progressive and consensus estimation in multihop sensor networks," IEEE Transactions on Signal Processing, vol. 59, no. 8, pp. 3863-3875, 2011.

[25] Z. Kuang, L. Li, J. Gao, L. Zhao, and A. Liu, "Partial offloading scheduling and power allocation for mobile edge computing systems," IEEE Internet of Things Journal, vol. 6, no. 4, pp. 6774-6785, 2019.

[26] J. Su, R. Xu, S. Yu, B. Wang, and J. Wang, "Idle slots skipped mechanism based tag identification algorithm with enhanced collision detection," KSII Transactions on Internet and Information Systems, vol. 14, no. 5, pp. 2294-2309, 2020.

[27] J. Su, R. Xu, S. Yu, B. Wang, and J. Wang, "Redundant rule detection for software-defined networking," KSII Transactions on Internet and Information Systems, vol. 14, no. 6, pp. 27352751, 2020. 